\title{
The Development of Expressive Drawing Abilities during Childhood and into Adolescence
}

\author{
Delphine Picard ${ }^{1,2}$ and Christophe Gauthier ${ }^{1,3}$ \\ ${ }^{1}$ Department of Psychology, Octogone-ECCD, University of Toulouse II, 5 allées Antonio Machado, 31058 Toulouse, France \\ ${ }^{2}$ Institut Universitaire de France, 75005 Paris, France \\ ${ }^{3}$ Department of Psychology, University of Nîmes, Site Vauban, Rue du Dr Salan, 30021 Nîmes, France
}

Correspondence should be addressed to Delphine Picard, delphine.picard@univ-tlse2.fr

Received 6 December 2011; Revised 9 March 2012; Accepted 10 March 2012

Academic Editor: Masha Gartstein

Copyright ( 2012 D. Picard and C. Gauthier. This is an open access article distributed under the Creative Commons Attribution License, which permits unrestricted use, distribution, and reproduction in any medium, provided the original work is properly cited.

\begin{abstract}
The way children portray emotions in their drawings of human and nonhuman topics is assumed to reflect their artistic, emotional, and cognitive development. This study was designed to investigate the development of expressive drawings during childhood and into adolescence, using a large age range (5-15 years) and sample size $(N=480)$, so as to provide a precise and comprehensive view of age-related changes in children's ability to produce expressive drawings. More specifically, we focused on children's developing ability to use the techniques of literal and metaphorical expression, either alone or in combination. We also examined the effects of sex, topics (house, tree, or person), and the depicted emotion (happiness or sadness) on the use of each expressive technique. The main findings were that there is a developmental shift between childhood (5-10 years) and adolescence (11-15 years) in the use of expressive techniques, from simple (literal) to more complex forms of expression (metaphorical).
\end{abstract}

\section{Introduction}

Drawing, one of children's favourite activities, has been studied from many different perspectives (e.g., changes in conceptual knowledge, motor skills, aspects of cognitive development, emotional states, or personality traits), thereby shedding light on various facets of children's psychological functioning (i.e., perceptual, motor, cognitive, emotional) [1]. Much research has been devoted to the analysis of the representational aspects of drawings, notably the degree of visual realism with which children depict reality. As discussed by Ives [2], "a drawing is much more than what it represents" (page 152). In addition to depicting the figurative aspects of everyday objects (e.g., a car), drawings can express psychological moods and basic emotions. For instance, the drawing of a person crying, and the drawing of a mutilated tree under a heavily hatched sky can both be expressive of sadness. In the former example, mood is expressed literally through facial features. In the latter example, it is expressed metaphorically through content (damaged object) and abstract (dark colors, heavy lines) cues.
Although it has for long been acknowledged that the way in which children express emotions in their drawings reflects their artistic, emotional, and cognitive development [3-5], expressive drawing has been surprisingly neglected in experimental investigation. In his recent book on children's drawings, Jolley [6] stated that "relative to the attention given to children's representational drawing, children's drawings as a means of communicating moods, feelings and ideas in an esthetic sense has very much been an under-researched area" (page 36). In the present study, we examined the development of children's and adolescents' ability to produce expressive drawings of human and nonhuman topics, as attested by their use of literal and/or metaphorical expression.

Broadly speaking, there have been two distinct and complementary approaches to the study of expressive drawing development. One approach examined artistic development from a subjective point of view, by measuring the perceived quality of expression in aesthetic drawings; the other approach examined expressive drawing development from a more objective or quantitative point of view, by quantifying 
the number of expressive cues in aesthetic drawings or by classifying the aesthetic drawings according to their expressive techniques. Our study pertains to the second approach and was designed primarily to provide a more precise and comprehensive picture of children's ability to produce expressive drawings than that currently offered by the research literature.

Within the first approach, there has been a debate in the literature regarding the existence of a U-shaped curve in the development of the (perceived) quality of expressive drawings. The claim that the quality of aesthetic drawings declines in middle childhood originated from the Harvard's project Zero team [7] with empirical support from Davis [8]. The U-shaped curve has been challenged empirically by several authors who revisited this model from a crosscultural perspective [9-11]. It appeared from these studies that the U-curve model of artistic development was bound by cultural assumptions and mostly reflected a modernistic view of art. Alternative developmental patterns were found, such a flat line pattern or even an inverted U-shaped pattern (antimodernistic view), depending on how drawings were evaluated by different cultures following their art values (see also [6] for further discussion on this debate).

Within the second approach, a body of research has been carried out on children's ability to make use of expressive cues and techniques in their drawings [2, 12-18]. These studies converge to suggest that children's expressive drawing improves with age. However, although highly informative, this corpus offers a fragmented overview of children's expressive drawing development, due to a large disparity in age ranges and methods across studies. Even though children of many different ages were observed: 4 years $[2,13]$, 5 years $[2,17], 6$ years $[13,14,18]$, 7 years $[2,12,14,15,17], 8$ years $[14], 9$ years $[2,13,14,16,18], 10$ years $[12,14,16], 11$ years $[2,15-17], 12$ years $[12,13,16,18], 13$ years $[2,16], 14$ years [16], 15 years [16], and 16 years [2], as were adults [2, 15, 17], none of these previous studies provided a comprehensive overview of the developmental changes that occur during childhood and into adolescence.

Studies also differed methodologically, as to the nature of the drawing task, the availability of color crayons, the type of topic to be illustrated, the use of a baseline drawing, and the analysis of the expressive drawings. With respect to task, most previous studies employed a drawing-fromimagination task, with children being given the explicit verbal instruction to produce expressive drawings [2, 13, 14, 16-18]. A single study used a drawing completion task [12]. Depending on the study, children were given color crayons to use as they wished $[13,14,16,17]$, or else they had no color crayons at all $[2,18]$. It is worth emphasizing that such between-studies variations, as restrictions on color use may alter children's inclination to use color as a metaphorical cue. With respect to topic, studies mostly involved drawings of trees $[2,12-14,16-18]$, houses $[13,15,16]$, or people $[14,15]$, but none of them tested all three topics together. In particular, despite the fact that all the studies contrasted positive and negative emotions (happy versus sad), only a few of them used a normal (neutral) drawing as a baseline to objectively detect changes introduced to make them express happiness or sadness [15-17]. Finally, the studies differed as to the way in which the expressive drawings were analyzed, with some studies focusing on the use of specific metaphorical cues such as size and color variations [17] and others classifying the drawings according to the expressive techniques [2, 15-17] or quantifying the number of metaphorical cues they contained $[13,18]$. As a result of all these disparities, no single comprehensive picture of the development of children's expressive abilities can be drawn from the currently available data.

To overcome these shortcomings, in the present study, we gathered data from a large sample of children and adolescents aged from 5 to 15 years who had to produce expressive drawings from their imagination. Using a withinparticipants design, we asked them to produce drawings of three familiar topics (a house, a tree, and a person) in three different versions (normal, happy, and sad). It is useful to study these three topics together as they pertain to several projective drawing tests $[19,20]$. In order to trace the developmental curves of expressive drawing abilities, we examined age-related changes in the children's and adolescents' use of literal and/or metaphorical expression. By classifying the drawings according to the basic expressive techniques employed, we were able to achieve a general qualitative framework for the different types of expressive cues that can be found in graphic productions (see Table 1 for further details of basic expressive techniques). We also assessed the effects of sex, topic (house, tree, person), and emotion (happiness, sadness) on the use of each expressive technique.

Our main hypotheses were as follows. Hypothesis 1 (agerelated trends): between childhood and adolescence, there would be a shift away from the use of literal expression toward the use of more complex forms of expressions, either a combination of literal and metaphorical techniques, or an exclusively metaphorical technique. We expected entry into adolescence (10-11 years) to be the pivotal period. The precise shapes of the developmental curves for each technique were examined. Hypothesis 2 (sex-related effects): if the sex-related effects observed in young adolescents [16] can be generalized to a wider developmental period, the girls in our study would display a greater ability to combine literal and metaphorical expressions and a lesser inclination to use literal expression alone than the boys, regardless of age.

\section{Method}

2.1. Participants. A total of 480 children and adolescents took part in the study. They were recruited from state schools in middle-class districts of southern French cities. The participants were divided into ten age groups ( 24 boys and 24 girls in each group): $5-6$ years $(n=48, M=5$ years 7 months, $\mathrm{SD}=3$ months), 6-7 years $(n=48, M=6$ years 7 months, SD $=4$ months), $7-8$ years $(n=48, M=7$ years 8 months, $\mathrm{SD}=4$ months), $8-9$ years $(n=48, M=8$ years 7 months, $\mathrm{SD}=6$ months $), 9-10$ years $(n=48, M=9$ years 5 months, $\mathrm{SD}=5$ months), $10-11$ years $(n=48, M=10$ years 6 months, $\mathrm{SD}=6$ months $), 11-12$ years $(n=48, M=11$ 
TABLE 1: Description of the basic expressive techniques.

\begin{tabular}{|c|c|}
\hline Expressive technique & Description \\
\hline Literal & $\begin{array}{l}\text { Expressive drawings featuring facial expression cues (e.g., happy face with a smile versus frowning face with } \\
\text { tears). Nonhuman topics are personified. }\end{array}$ \\
\hline Metaphorical & $\begin{array}{l}\text { Expressive drawings featuring abstract and/or content cues. "Abstract cues" refer to changes in the formal } \\
\text { properties of the drawings, such as lines (e.g., light, thin versus dark, heavy), color (e.g., warm and bright versus } \\
\text { cold and dark), and size (e.g., oversized versus undersized). "Content cues" refer to changes in the figurative } \\
\text { aspects of the drawings, such as weather (e.g., sunshine versus clouds, storms, and rain), season (e.g., spring, } \\
\text { flowers versus winter, fallen leaves, absent vegetation), state (e.g., radiant versus damaged or mutilated object), } \\
\text { and sociability (e.g., people playing, animals, gifts versus loneliness, empty surroundings). }\end{array}$ \\
\hline
\end{tabular}

years 5 months, $\mathrm{SD}=4$ months $), 12-13$ years $(n=48$, $M=12$ years 6 months, $\mathrm{SD}=5$ months), 13-14 years ( $n=48, M=13$ years 7 months, SD $=5$ months), and 1415 years ( $n=48, M=14$ years 6 months, $\mathrm{SD}=5$ months). None of them were known to suffer from a mood disorder, a psychomotor drawing, or handwriting disorder. Written parental consent was obtained for each participant, and the study was carried out in accordance with the Declaration of Helsinki sixth revision, 2008.

2.2. Materials. The materials consisted of white sheets of paper, a standard writing pencil, and nine crayons (black, brown, green, blue, red, purple, orange, yellow, and pink).

2.3. Procedure. The session started with a warm-up exercise during which all participants completed the parallel lines test. In this figural test of divergent thinking [21], participants had to produce as many different drawings as possible using two parallel lines as their starting point. This task was used to elicit figural fluency and creative behavior in the participants before they performed the expressive drawing task. In the expressive drawing task, participants had to produce drawings of three different topics (house, tree, and person) in three different versions (normal first, then happy, and sad). Note that we choose not to ask children to draw free (happy/sad) pictures. Close inspection of the data obtained by Jolley et al. [13] with free pictures versus imposed topics revealed that children obtained similar quantitative scores of expressive drawing with both types of tasks. We therefore believe that our task does not restrict children's opportunity to draw expressively and is a relevant task to the study of children's art. The order of presentation of the three topics and the two emotions was counterbalanced across participants of each sex and age group. Participants were allowed to use the pencil and the nine color crayons as they wished. For each expressive drawing, participants were asked to justify the changes they had made in order to express the target emotion. All verbal justifications were transcribed by the experimenter. Time for drawing the nine expressive drawings was not limited, and children were free to have rests if necessary during the completion of the expressive drawing task. The full session (warm-up exercise plus nine drawings) lasted 30 minutes per child on average. Participants were thanked and complimented on their drawings.
2.4. Coding of the Drawings. The happy and sad drawings were coded according to their expressive technique: "literal," "literal and metaphorical," or "metaphorical." These three mutually exclusive expressive techniques are described in Table 1. Drawings where few or no modifications had been made to express emotion were classified as "nonexpressive." The coding was performed by a trained judge ( $\mathrm{PhD}$ student), who used the normal drawings as reference (baseline) drawings to detect any changes. Coding relied on the children's verbal explanations as to how they had made their drawings look happy or sad for confirmatory purpose only. A second expert judge coded a random sample of drawings (20\%). Interrater reliability was high (Cohen's kappa coefficient $=$ $0.98, P<0.05)$.

\section{Results}

Table 2 summarizes the main results. Figure 1 shows the developmental curves for each drawing technique. A mixed analysis of variance (ANOVA) was run on the frequency of occurrence of each drawing technique with age (10), sex (2) as between-participants variables and topic (3), emotion (2) as within-participants factors. Although it may seem unconventional to use parametric tests with binary data, some authors (e.g., $[22,23]$ ) have shown that ANOVAs can be performed with such data under certain conditions (e.g., the number of degrees of freedom of the error term must be above 40), which were satisfied here. Because four separate ANOVAs were run on the data (one per drawing technique), we used an alpha level of 0.05 which was adjusted to 0.0125 after Bonferroni correction. We report partial eta squared $\left(\eta_{P}^{2}\right)$ as measure of effect size. Significant effects only will be reported in Section 3.

\subsection{Developmental Curves of Expressive Drawing Abilities.} First, only a minority of the children (no more than $10 \%$ of the youngest children) failed to meet the requirements to draw expressively. As can be seen from Figure 1, nonexpressive drawings tended to disappear from age 7 onwards. Age therefore induced a quick decline in the production of non-expressive drawings, as attested by a significant main effect of age, $F(9,460)=3.76, P<0.001$, with a small effect size, $\eta_{P}^{2}=0.07$. Tukey's HSD post hoc tests showed a significant decline in the occurrence of non-expressive 
TABle 2: Distribution (in percentage) of the drawings according to their expressive techniques by age, sex, topic, and emotion. The occurrence (in percentage) of nonexpressive drawings is shown in italics.

\begin{tabular}{|c|c|c|c|c|c|c|}
\hline & & Nonexpressive & Literal & Literal and metaphorical & Metaphorical & Total \\
\hline \multirow{10}{*}{ Age } & $5-6$ yrs & 9 & 56 & 20 & 15 & 100 \\
\hline & $6-7$ yrs & 6 & 52 & 21 & 20 & 100 \\
\hline & $7-8$ yrs & 2 & 46 & 32 & 20 & 100 \\
\hline & $8-9$ yrs & 2 & 30 & 48 & 20 & 100 \\
\hline & $9-10$ yrs & 2 & 25 & 41 & 32 & 100 \\
\hline & $10-11$ yrs & 0 & 16 & 55 & 29 & 100 \\
\hline & $11-12$ yrs & 0 & 28 & 43 & 28 & 100 \\
\hline & $12-13$ yrs & 1 & 25 & 36 & 38 & 100 \\
\hline & $13-14$ yrs & 1 & 22 & 35 & 42 & 100 \\
\hline & $14-15 \mathrm{yrs}$ & 2 & 23 & 38 & 38 & 100 \\
\hline \multirow{2}{*}{ Sex } & Boys & 3 & 36 & 33 & 28 & 100 \\
\hline & Girls & 2 & 29 & 41 & 29 & 100 \\
\hline \multirow{3}{*}{ Topic } & House & 3 & 23 & 32 & 42 & 100 \\
\hline & Tree & 3 & 23 & 32 & 42 & 100 \\
\hline & Person & 1 & 52 & 46 & 1 & 100 \\
\hline \multirow{2}{*}{ Emotion } & Happy & 3 & 34 & 37 & 26 & 100 \\
\hline & Sad & 2 & 30 & 37 & 31 & 100 \\
\hline
\end{tabular}

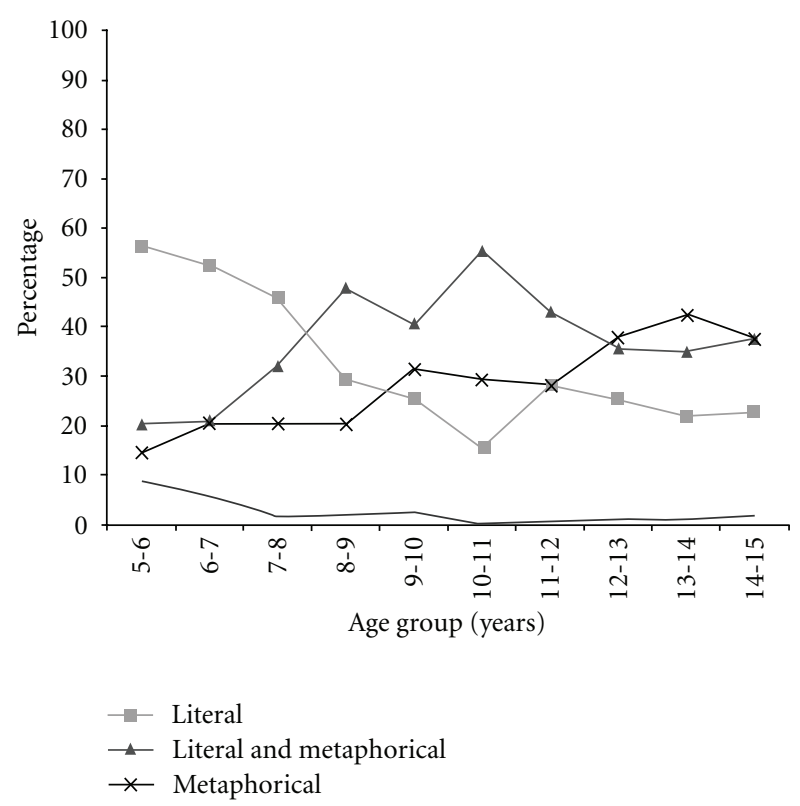

FIgURE 1: Age-related changes in the distribution (in percentage) of drawings according to expressive techniques. The plain line indicates the developmental curve for non-expressive drawings.

drawings between 5-6 and 7-8 years of age $(P<0.01)$, with further significant between-group differences beyond age 8 .

Second, the dominant expressive technique used by the young children was literal expression. This technique also declined with age, as attested by a significant main effect of age, $F(9,460)=12.05, P<0.001$, with a medium effect size, $\eta_{P}^{2}=0.19$. As illustrated in Figure 1, and in line with Hypothesis 1, there was a pronounced linear decline in literal expression between $5-6$ years $(56 \%)$ and $10-11$ years $(16 \%)$, the age at which it reached its lowest level. Tukey's HSD post hoc tests indicated a significant decline in the occurrence of literal expression between 5-6 and 10-11 years of age $(P<$ $0.01)$, with no further significant between-group differences beyond age 11 . The developmental curve of literal expression thus featured two phases: a decline during childhood (510 years), followed by a plateau during adolescence (11-15 years).

Third, as a counterpoint to the decrease in literal expression, children displayed an increasing ability to combine literal and metaphorical expressions in their drawings as they grew older. The ANOVA yielded a significant main effect of Age on the occurrence of combined literal and metaphorical expression, $F(9,460)=6.30, P<0.001$, with a medium effect size, $\eta_{P}^{2}=0.11$. In line with Hypothesis 1 , a marked increase in the use of literal and metaphorical expression occurred between 5-6 years (20\%) and 10-11 years $(55 \%)$, the age at which it peaked. Interestingly, we noted a subsequent brief decline in the use of this combined technique between 10-11 years and 12-13 years, followed by a period of stagnation up to age 14-15. Tukey's HSD post hoc tests also indicated a significant increase in the occurrence of literal and metaphorical expression between 5 6 and $10-11$ years of age $(P<0.01)$, followed by a decrease between $10-11$ years and $12-13$ years $(P<0.05)$, with no 
further significant between-group differences up to 14-15 years. The developmental curve of literal and metaphorical expression thus featured three phases: an initial increase during childhood (5-10 years), a brief intermediate decline in early adolescence (11-13 years), and a final plateau (13-15 years).

Fourth and finally, both children and young adolescents displayed a limited use of metaphorical expression: from 56 to $8-9$ years, use of this technique did not exceed $20 \%$. Thereafter, it slowly increased to $40 \%$ at $13-14$ years, where it remained the dominant expressive technique, along with literal and metaphorical expression (see Figure 1). Age had a significant impact on the occurrence of metaphorical expression, $F(9,460)=5.49, P<0.001$, with a medium effect size, $\eta^{2}{ }_{P}=0.10$. Tukey's HSD post hoc tests failed to reveal any significant variations in the occurrence of metaphorical expression before age 8-9. The main increase in the occurrence of metaphorical expression occurred between 8-9 and 13-14 years $(P<0.01)$. The developmental curve of metaphorical expression thus featured two main phases: an initial plateau during childhood (5-9 years), followed by a slow increase starting at the end of childhood and continuing during adolescence (9-15 years). It should be noted that this developmental curve was concerned only with the expressive drawings of the nonhuman topics. Indeed, metaphorical expression alone was quite never involved in the drawing of the human topic whatever the age. As a result, a 2-way interaction effect between age and topic was found on the frequency of metaphorical expression, $F(18,920)=3.83$, $P<0.001, \eta^{2}{ }_{P}=0.07$. This higher order effect indicated that there was age-related increment of metaphorical expression for both the expressive drawings of the house and the tree (as described above) and a flat line pattern for the expressive drawing of the person (constant minimal use of metaphorical expression).

3.2. Sex-Related Differences. The ANOVAs yielded significant main effects of sex on the frequency of literal expression, $F(1,460)=6.71, P<0.01$, with a small effect size, $\eta^{2}{ }_{P}=0.02$ and literal and metaphorical expression, $F(1,460)=7.42$, $P<0.01$, with a small effect size, $\eta^{2}{ }_{P}=0.02$. As can be seen from Table 2, boys used literal expression alone (36\%) more often than girls did (29\%). Conversely, girls combined literal and metaphorical expressions (41\%) more often than boys $\operatorname{did}(33 \%)$. This pattern of results fits in with Hypothesis 2.

3.3. Topic Effects. Main effects of topic were found on the frequency of literal expression, $F(2,920)=139.49, P<$ 0.0001 , with a medium effect size, $\eta_{P}^{2}=0.23$, literal and metaphorical expression, $F(2,920)=22.90, P<0.001$, with a small effect size, $\eta^{2}{ }_{P}=0.05$, and metaphorical expression, $F(2,920)=285.34, P<0.001$, with a large effect size, $\eta^{2}{ }_{P}=$ 0.38 . As can be seen from Table 2, metaphorical expression was involved far more in the drawing of nonhumans topics (house, tree: $42 \%$ ) than in the drawing of the human topics (1\%). Expressive drawings of people elicited literal expression (52\%) and literal and metaphorical expression $(46 \%)$ more often than drawings of nonhuman topics (house and tree taken together: $23 \%$ for literal expression and 32\% for literal and metaphorical expression). Tukey's HSD post hoc tests indicated significant differences in the occurrence of each expressive technique (literal, metaphorical, literal and metaphorical) between drawings of nonhuman topics and drawings of people (all $P s<0.01$ ), with no significant differences between drawings of houses and trees.

3.4. Emotion Effects. We found emotion caused slight qualitative differences in two of the expressive techniques used for drawing happy and sad topics. Namely, literal expression was used more often in happy drawings (34\%) than in sad ones $(30 \%)$, as revealed by a significant main effect of emotion on the frequency of use of literal expression, $F(1,460)=12.65$, $P<0.001$, with a small effect size, $\eta^{2}{ }_{P}=0.03$. By contrast, sadness elicited metaphorical expression more often $(31 \%)$ than happiness did $(26 \%)$, as revealed by a significant main effect of emotion on the frequency of use of this technique, $F(1,460)=38.38, P<0.001$, with a small effect size, $\eta_{P}^{2}=0.08$. Again, it should be noted that the emotion effect observed for metaphorical expression was concerned only with the expressive drawings of the nonhuman topics. A 2-way interaction effect between emotion and topic on the frequency of metaphorical expression, $F(2,920)=11.22$, $P<0.001, \eta_{P}^{2}=0.02$, indicated that sadness elicited metaphorical expression more often than happiness did for both the house and the tree but not for the person (because metaphorical expression was quite never involved in drawings of the person).

\section{Discussion}

This study was intended to provide an overview of the development of expressive drawing abilities from childhood to early adolescence. We found that children as young as 7 years were able to produce expressive drawings of familiar topics with a very low failure rate (less than $2 \%$ ) when specifically asked to do so, and even below that age, only a small percentage of children failed on the task (10\%), suggesting that the ability to draw expressively emerges quite early in the course of children's drawing development (see also $[2,13]$ ). As expected (Hypothesis 1), we found clear agerelated changes in the types of techniques used by children to draw expressively, with entry into adolescence being a pivotal period.

Expressive drawings typical of the childhood period (5-10 years) were initially characterized by the dominant use of a simple expressive technique, referred to here as "literal expression." Literal expression characterised most of the drawings produced by the youngest children (5-6 years). However, as the children grew older, this simple expressive technique declined markedly and was replaced by the comparatively more complex technique of "literal and metaphorical expression." This combined expressive technique became increasingly prominent during childhood, coming to represent the dominant technique by the end of childhood. Expressive drawings typical of the adolescent period (11-15 years) were initially characterized by the 
predominant use of literal and metaphorical expressions. However, as adolescents grew older, they made less use of this technique and displayed an increasing inclination to use the sophisticated technique of "metaphorical expression" on its own. Metaphorical expression became increasingly frequent in the course of adolescence, eventually coming to represent one of the two preferred expressive techniques at age 15 (metaphorical expression, literal and metaphorical expression). There was thus a shift between childhood and adolescence in the use of expressive techniques, from simple (literal) to more complex forms of expression (literal and metaphorical expression, metaphorical expression alone).

As expected (Hypothesis 2), we found sex-related differences in the use of expressive techniques. Extending previous results [16], our study revealed that, during both childhood and early adolescence, girls demonstrate a greater ability to combine literal and metaphorical expression in their drawings and a lesser inclination to use literal expression alone than boys. Interestingly, these sex-related effects did not interact with age, suggesting that there are some stable characteristics that differentiate expressive drawings produced by boys from those produced by girls. Picard and Boulhais [16] have argued that these sex differences "are consistent with the idea that boys and girls differ in emotional expressivity" (page 3), and that girls' "greater ease in the nonverbal communication of emotional states may extend to the artistic domain" (page 4). The issue of whether these sex-related differences result from biological or psychocultural differences in the development of boys and girls is still open to debate (see, for example, [24, 25]).

Drawing different topics were found to call for the use of different types of expressive drawing techniques. Namely, our findings indicated that whereas expressive drawings of people mostly called for literal expression, drawings of nonhuman topics (house, tree) preferentially triggered more complex expressive techniques, either including or restricted to metaphorical expression. In particular, metaphorical expression was almost never used alone in expressive drawings of people. These findings are congruent with previous results in the research literature (see $[2,15])$. In addition, we found that the valence of the to-be-depicted emotion had a slight effect on the type of expressive technique employed by the children. Our study revealed that, in contrast to happiness, sadness elicited metaphorical expression more often than literal expression. This is a new finding, which suggests that metaphorical expression may be particularly suited to connoting sadness in the visual arts, as evidenced in paintings from the Romantic period (see, for example, the melancholy landscapes by the German painter C. D. Friedrich).

To conclude, the present study yielded descriptive accounts of the normal developmental curves of expressive drawing abilities during childhood and early adolescence. It additionally highlighted differences in the use of literal and metaphorical expression according to the participants' sex, the type of topic to be illustrated, and the valence of the emotion being expressed in the drawing. Taken together, these findings add to our understanding of expressive drawing development and the factors that affect children's and young adolescents' ability to draw expressively. They also provide useful baseline data for future comparative studies in developmental psychopathology. For instance, the issue of whether expressive drawings produced by children with autism spectrum disorder (ASD) reflect a broadly similar developmental pattern to that found in typically developing children, or whether there are qualitative differences between the two populations, is a sensitive question. Children with ASD have been described as having considerable difficulty understanding nonliteral forms of communication (metaphors, ironic statements, and figurative language), showing a tendency to interpret these forms literally [26]. Children with ASD are also known to experience difficulty in the emotional domain, especially in the processing of facial expressions of emotions $[27,28]$. Therefore, we would expect impairments or specificities in the production of expressive/metaphoric drawings in children with ASD compared with normal children. However, few studies so far have been designed to assess between-populations differences in the domain of expressive drawing (see [29] for a very recent study with children with ASD), partly due to the lack of available baseline data regarding normal expressive drawing development. Accordingly, the data collected in the present study should prove useful in future research.

\section{Acknowledgments}

This paper was supported by a grant from the Fondation de France "Research on Cognitive and Emotional Development in Children." The authors are grateful to the parents, teachers, and children from the participating schools. They also wish to thank E. Wiles-Portier for her helpful comments on the paper and the psychology students of Nîmes University for their assistance in collecting data.

\section{References}

[1] C. Lange-Küttner and A. Vinter, Eds., Drawing and the NonVerbal Mind, Cambridge University Press, Cambridge, UK, 2008.

[2] S. W. Ives, "The development of expressivity in drawing," British Journal of Educational Psychology, vol. 54, pp. 152-159, 1984.

[3] E. H. Gombrich, "The visual image," Scientific American, vol. 227, no. 3, pp. 82-96, 1972.

[4] N. Goodman, Languages of Art, Bobbs-Merrill, Indianapolis, India, 1968.

[5] M. J. Parsons, How we Understand Art: A Cognitive Developmental Account of Aesthetic Experience, Cambridge University Press, Cambridge, UK, 1987.

[6] R. Jolley, Children and Pictures: Drawing and Understanding, Blackwell, Malden, Mass, USA, 2010.

[7] H. Gardner and E. Winner, "First intimations of artistry," in U-Shaped Behavioural Growth, S. Strauss, Ed., Academic Press, New York, NY, USA, 1982.

[8] J. H. Davis, "Drawing's demise: U-shaped development in graphic symbolisation," Studies in Art Education, vol. 38, pp. 132-157, 1997.

[9] F. Haanstra, M.-L. Damen, and M. van Hoorn, "The U-shaped curve in the low countries: a replication study," Visual Arts Research, vol. 37, pp. 16-29, 2011. 
[10] A. M. Kindler, "From the U-curve to dragons: culture and understanding of artistic development," Visual Arts Research, vol. 26, pp. 15-28, 2000.

[11] D. Pariser and A. van den Berg, "The mind of the beholder: some provisional doubts about the U-curve aesthetic development thesis," Studies in Art Education, vol. 38, pp. 158-178, 1997.

[12] T. Carothers and H. Gardner, "When children's drawings become art: the emergence of aesthetic production and perception," Developmental Psychology, vol. 15, no. 5, pp. 570 $580,1979$.

[13] R. P. Jolley, K. Fenn, and L. Jones, "The development of children's expressive drawing," British Journal of Developmental Psychology, vol. 22, no. 4, pp. 545-567, 2004.

[14] S. Morra, B. Caloni, and R. d'Amico, "Working memory and the intentional depiction of emotions," Archives de Psychologie, vol. 62, pp. 71-78, 1994.

[15] D. Picard, C. Brechet, and R. Baldy, "Expressive strategies in drawing are related to age and topic," Journal of Nonverbal Behavior, vol. 31, no. 4, pp. 243-257, 2007.

[16] D. Picard and M. Boulhais, "Sex differences in expressive drawing," Personality and Individual Differences, vol. 51, pp. 850-855, 2011.

[17] D. Picard and S. Lebaz, "Symbolic use of size and color in freehand drawing of the tree: myth or reality?" Journal of Personality Assessment, vol. 92, no. 2, pp. 186-188, 2010.

[18] A. S. Winston, B. Kenyon, J. Stewardson, and T. Lepine, "Children's sensitivity to expression of emotion in drawings," Visual Art Research, vol. 21, pp. 1-15, 1995.

[19] J. N. Buch, "The HTP test," Journal of Clinical Psychology, vol. 4, pp. 151-159, 1948.

[20] K. Machover, Personality Projection in the Drawings of the Human Figure, Charles Thomas, Springfield, Ill, USA, 1949.

[21] E. P. Torrance, The Torrance Tests of Creative Thinking, Personnel Press, Princeton, NJ, USA, 1966.

[22] T. Greer and W. P. Dunlap, "Analysis of variance with ipsative measures," Psychological Methods, vol. 2, no. 2, pp. 200-207, 1997.

[23] G. H. Lunney, "Using analysis of variance with a dichotomous dependent variable: an empirical study," Journal of Educational Measurement, vol. 7, pp. 239-263, 1970.

[24] K. Flannery and M. Watson, "Sex differences and gender role differences in children's drawings," Studies in Art Education, vol. 36, pp. 114-122, 1995.

[25] M. Iijima, O. Arisaka, F. Minamoto, and Y. Arai, "Sex differences in children's free drawings: a study on girls with congenital adrenal hyperplasia," Hormones and Behavior, vol. 40, no. 2, pp. 99-104, 2001.

[26] F. G. Happé, "The role of age and verbal ability in the theory of mind task performance of subjects with autism.," Child Development, vol. 66, no. 3, pp. 843-855, 1995.

[27] M. Balconi and A. Carrera, "Emotional representation in facial expression and script. A comparison between normal and autistic children," Research in Developmental Disabilities, vol. 28, no. 4, pp. 409-422, 2007.

[28] G. Celani, M. W. Battacchi, and L. Arcidiacono, "The understanding of the emotional meaning of facial expressions in people with autism," Journal of Autism and Developmental Disorders, vol. 29, no. 1, pp. 57-66, 1999.

[29] R. P. Jolley, R. O’Kelly, and C. M. Barlow, “The development of expressive drawing in children with autism," in preparation. 


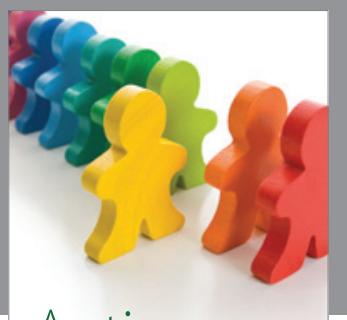

Autism

Research and Treatment
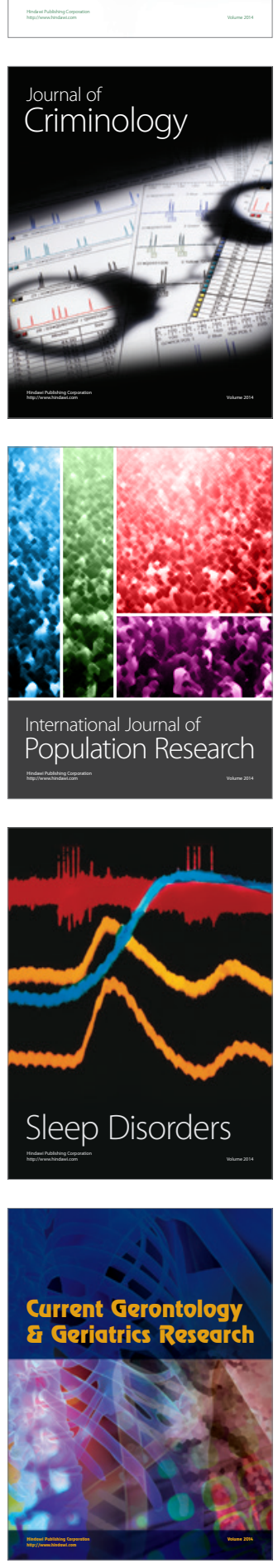
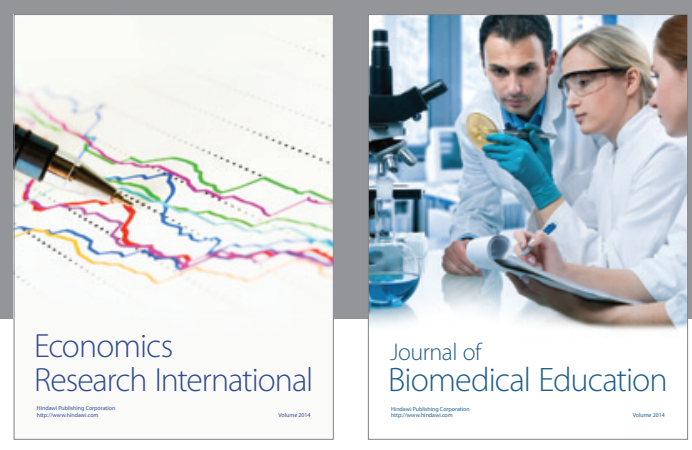

Journal of

Biomedical Education

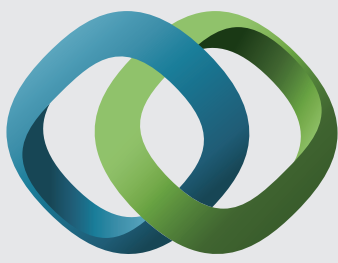

\section{Hindawi}

Submit your manuscripts at

http://www.hindawi.com
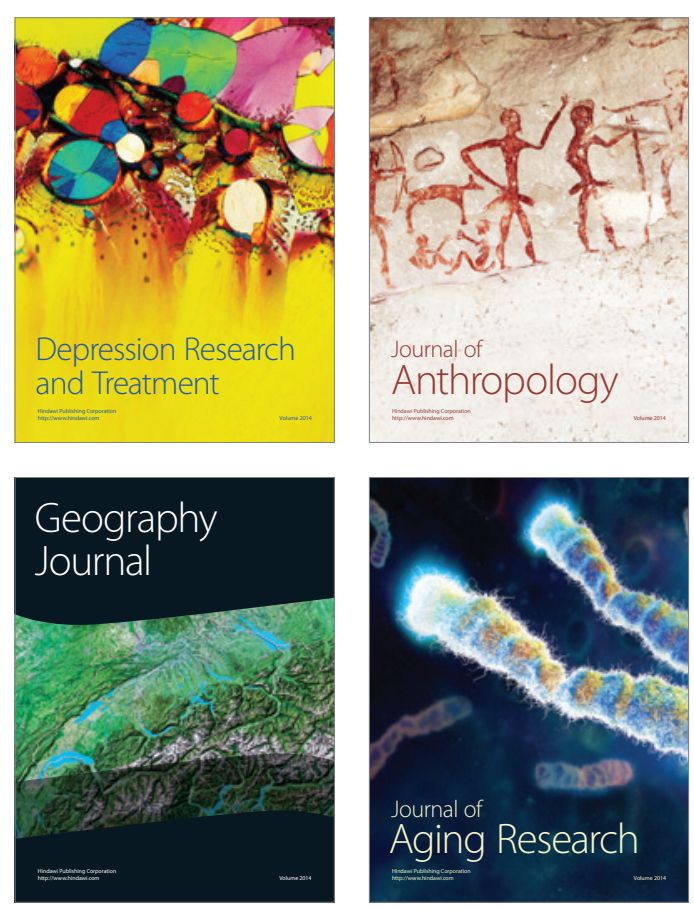

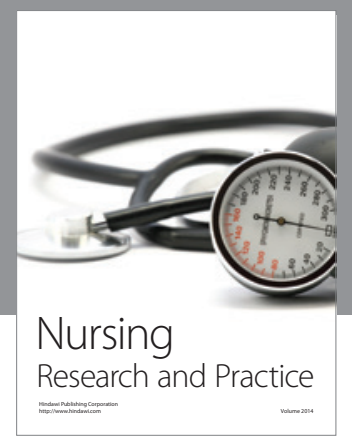

Nursing

Research and Practice

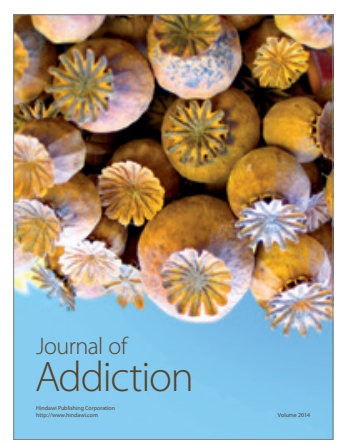

Child Development

Research

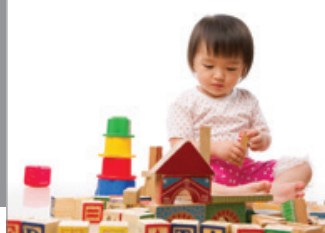

迥
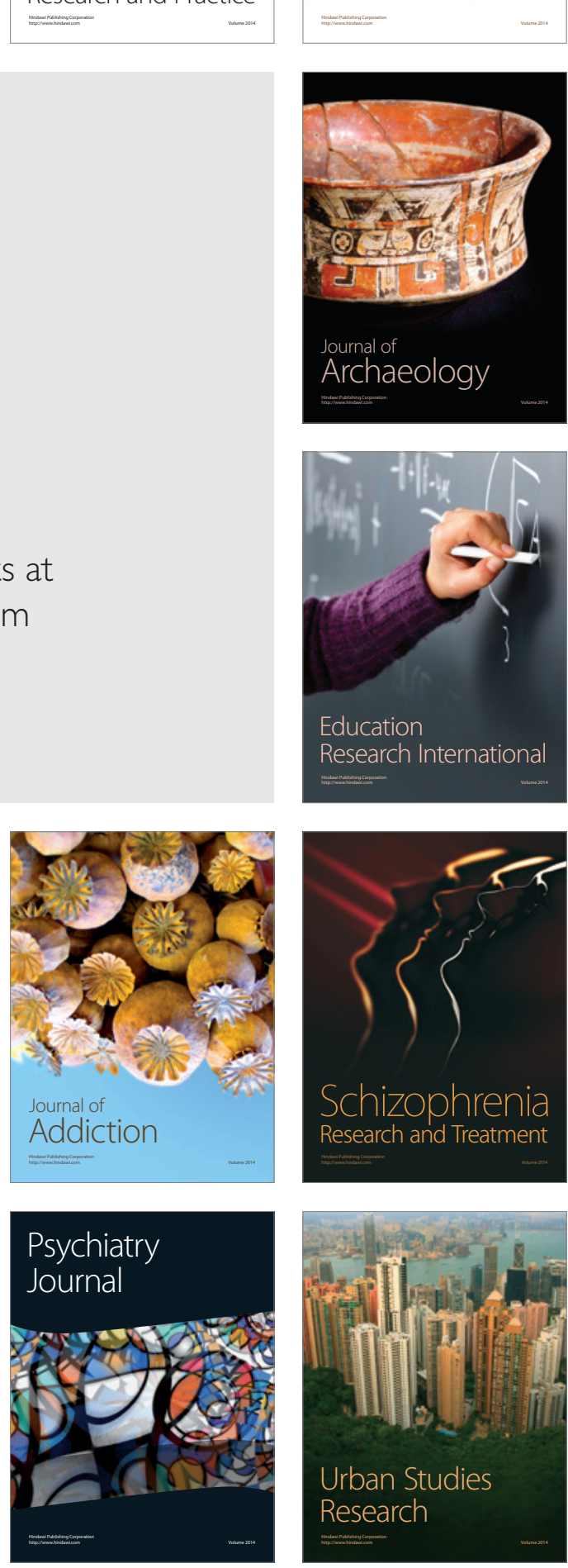